\title{
A taxonomy of : The reviews on Cloud Computing Direction (CCD)
}

\author{
Alla Talal Yassin*, Ban Kassim Alnidawy \\ UOITC, Ministry of Higher Education and Scientific Research Baghdad, Iraq \\ *Corresponding author E-mail: allatalal@yahoo.com
}

\begin{abstract}
Copyright $\odot$ 2015Dr.Alla Talal Yassin, Ban Kassim Alnidawy. This is an open access article distributed under the Creative Commons Attribution License, which
\end{abstract} permits unrestricted use, distribution, and reproduction in any medium, provided the original work is properly cited.

\begin{abstract}
The manufacturing industry is undergoing a major transformation enabled by IT and related smart technologies. The main thrust of Cloud computing is to provide on-demand computing services with high reliability, scalability and availability in a distributed environment. This paper summarizes our taxonomy of the CC review direction. The goals of this taxonomy were (i) clarify the needs and the directions of the use of the CC, (ii) define the academic and practical issues involved in CC, (iii) learn the state of the directions on methodologies of the CC, (iv) identify future research directions, which benefit the short and long terms. The taxonomy has concluded that (i) CC is advantageous in dealing with changes and uncertainties in the every-changing environment. (ii)It has been found that few existing CC can achieve the objective of security. (iii) The obstacles of the development of CC include the difficulties to identify and generalize the requirement of CC security, the lake of effective technologies that can be used to support the clouding use, and no international origination that serves for standardizing the modular components for cloud computing processes. In this paper, we use the IVSL(The Iraq Virtual Science Library) to select the free, full-text access to papers from major publishers as well as a large collection of on-line educational materials.
\end{abstract}

Keywords: Cloud Services; Cloud Computing; Survey

\section{Introduction}

Cloud computing is a general term for anything that involves delivering hosted services over the Internet.

A cloud service has three distinct characteristics that differentiate it from traditional hosting. It is sold on demand, typically by the minute or the hour; it is elastic -- a user can have as much or as little of a service as they want at any given time; and the service is fully managed by the provider (the consumer needs nothing but a personal computer and Internet access).

Significant innovations in virtualization and distributed computing, as well as improved access to high-speed Internet and a weak economy, have accelerated interest in cloud computing.

\section{A taxonomy of $\mathrm{CC}$}

This paragraph, clarify the needs and the directions of the use of the CC. Mondale suggests a stochastic hill which has been used for load distribution in Cloud computing environment [1]. The soft computing based approach has been compared with two approaches Round Robin first came first serve.

The rustles are quite encouraging. However, uses of other soft computing techniques are needed to be studied for further improvement [2]. Cheng describes a development of technology often, which will need to consider the possible impact within the legal infrastructure. Sometimes the possible impact will create a whole new legal issue. Sometimes it will make a preexisting legal issue prominent [3].

Lin studies the resource allocation at the application level, instead of studying how to map the physical resources to virtual resources for better resource utilization in cloud computing environment. He also studies the need to manage the applications in cloud computing creates the challenge of on-demand resource provisioning and allocation in response to dynamically changing workloads [4]. 
In the otherwise "Sun" mention that high security is one of the major obstacles for opening up the new era of the long dreamed vision of computing as a utility. As the sensitive applications and data are moved into the cloud data centers, run on virtual computing resources in the form of virtual machine. These unique attributes, however, poses many novel security challenges such as accessibility vulnerabilities, virtualization vulnerabilities, and web application vulnerabilities. With advancement of cloud computing and increasing number of cloud user, security, and privacy and trust dimensions will continuously increase. He also said that to protect private and sensitive data that are processed in data centers, the cloud user needs to verify (a) the real exists within the cloud computing environment in the world; (c) the security of information in the cloud; and (b) the trustworthiness of the systems in cloud computing environment [5]. Tao describes a generic framework implemented for Cloud users to access the various Cloud infrastructures in a unified fashion. The framework provides a graphical interface where service requests can be described and submitted, and services can be executed or terminated. The framework also enables the interconnection of different Cloud platforms. Currently, we have developed core architecture with basic, Cloud independent interfaces and views. This architecture can be easily extended to connect any Cloud infrastructure [6].

Kuyucu discourse both concepts; cost and energy, are the keywords for sustainable development and a well fair functioning economy. What cloud system offers simply signifies simplifying the machinery and outsourcing all that is possible in accordance with the need of the consumer. Cloud computing cannot be a total transfer of all hardware and software but offers a choice to be made, which hardware and software should be kept and what should not be [7].

Choo describes Cloud computing as a subject to frequent attacks by cyber criminals, who may be able to hijack and use them for criminal purposes, hence, adding to the challenge of growing volumes of digital evidence in each specific case under investigation. In addition, cloud services can be used as a launching pad for new attacks or to store and distribute criminal data by cyber criminals, organized-crime groups and politically-motivated actors to avoid the scrutiny of law enforcement and national-security agencies [8]. Gaofeng mentioned that the use of cloud computing by criminals means that their devices will be virtualized, geographically distributed and ephemeral, presenting technical and jurisdictional challenges for their identification and seizure by law enforcement and national-security agencies. These can impede digital forensic investigators and potentially prevent law enforcement and national-security agencies from acquiring digital evidence and analyzing digital content forensically in a timely fashion [9].

Other researchers such as Mell and Grance also mention that the sourcing and delivery model for enabling convenient, on-demand network access to a shared pool of configurable computing resources that can be rapidly provisioned and released with minimal management effort or service provider interaction infrastructure as a Service is a basic product, which can be offered by a cloud provider. It typically allows a cloud computing user to provision infrastructure on which they can run any software of their choosing [10].

Chow describes Cloud data security, the trade-off, here is the additional performance hit that is incurred during data access and so this is by no means universally employed by cloud providers. A secondary issue is in attackers gaining it is always a sound strategy to ensure that copies of critical data are securely replicated and stored in alternative physical locations to ensure that attacks/losses are mitigated but, in this case, special care must be taken to address legal and security concerns[11], [12], [13].

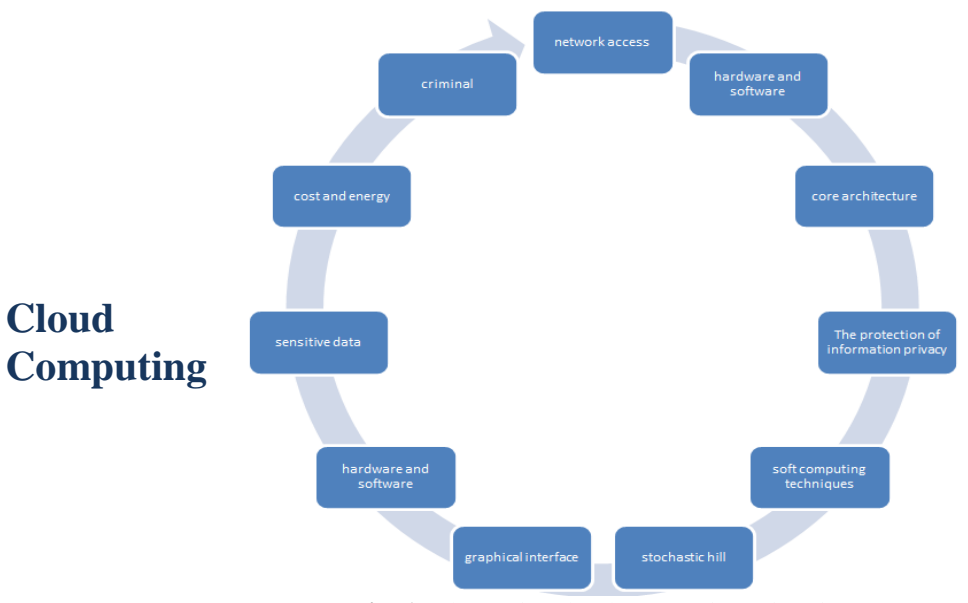

Fig. 1: The Main Cloud Computing Direction

A survey on security issues in service delivery models of cloud computing has been done from many researchers. Security issues in service models was a direction on cloud computing utilizes and have three delivery models by which different types of services are delivered to the end user. The three delivery models are the SaaS, PaaS and IaaS which provide infrastructure resources, application platform and software as services to the consumer[14], [15].

Many other directions were found like data, Network security, data locality, integrity, segregation, access, authentication, confidentiality, web application security, breaches, Virtualization vulnerability, availability, identity management and sign-on process, autonomic computing systems in [16], [17], [18], [19]. 
While artificial intelligent in [20], [21], [22], [38], and [43].Cloud computing is a disruptive technology with profound implications not only for Internet services but also for the IT sector as a whole. Still, several outstanding issues exist, particularly related to service-level agreements (SLA), security and privacy, and power efficiency[22], [23], and [24]. As described in the paper, currently security has a lot of loose ends, which scares away a lot of potential users. Until a proper security, module is not in place, potential users will not be able to leverage the advantages of this technology. This security module should cater to all the issues arising from all directions of the cloud. Until then, cloud environment will remain cloudy. Nabil Sultan, Cloud computing for education[25], [37].Security-oriented cloud computing platform for critical infrastructures, and hacking attacks with data loss or corruption has been mentioned in [25]. Mobile cloud computing a survey on motivation for a mobile cloud, Image processing, natural language processing, sharing GPS/Internet data, Sensor data applications, multimedia search, Social networking. A taxonomy of mobile cloud computing: Operational level issues, End user level issues, Service and application level issues, Privacy, security and trust, Context-awareness, Data's management, Cost-benefit analysis: It is important to analyze the costs of offloading on to the cloud such as time, energy and monetary, versus monolithic execution / storage beforehand [26,27,28]. The cloud user puts the cloud provider in charge of maintaining the leased virtual computers and software as well as of configuring the server farm, thus freeing their IT staff from these jobs and enabling them to focus on the development of IT innovations and business applications support[29-36].Smart manufacturing with cloud computing cloud computing can be effective in offering Business-to-business (B2B) solutions for commerce transactions between businesses, such as between a manufacturer and a wholesaler, or between a wholesaler and a retailer. Cloud-based solutions enable bettering - grated and more efficient processes. Internet-based manufacturing or distributed manufacturing virtual enterprise and distributed manufacturing A comprehensive data warehouse is utilized to store CNC manufacturing information with STEP-NC data model utilized as the basis for representing manufacturing knowledge that is augmented with XML schema [38-42].Cost effectiveness of commercial computing clouds. A costeffective cloud computing framework for accelerating multimedia communication simulations the performance of the proposed systems has to be evaluated in many different network scenarios and for several values of all the key parameters[43-46]. This work focused on investigating the cost-performance tradeoff of a cloud computing approach to run simulations frequently encountered during the research and development phase of multimedia communication techniques, characterized by several develop-simulate-reconfigure cycles[47-56].A framework for ranking of cloud computing services Cloud computing has emerged as a paradigm to deliver on demand resources, platform, software to customers similar to other utilities[57-60].The growths of public Cloud offerings, for Cloud customers it has become increasingly difficult to decide, which provider can fulfill their requirements[60-72]. Currently most of these existing methods focused on the optimization of allocating physical resources to their associated virtual resources and migrating virtual machines to achieve load balance and increase resource utilization. These methods require the suspension of the cloud computing applications due to the mandatory shutdown of the associated virtual machines[73-81]. The protection of information privacy under the development of cloud computing technology is the latter situation[82-89]. Every element in the cloud should be analyzed at the macro and micro level, and an integrated solution must be designed and deployed in the cloud to attract and enthrall the potential consumers [90-100].Access to the data through compromising shared resources, authorization, accounting and user control: virtual machines or services operated in the cloud should still be subject to the same hardening as with traditional systems [101-107].

\section{Statistical Methodology}

Our sample articles were (107) randomly selected from the period 1998-2013; the only determination was the key search which was "cloud computing." Table (1) shows the statistical distribution for the directions.

Table 1: The Statistical Distribution for the Directions

\begin{tabular}{|l|l|l|}
\hline Direction & No. & Per. \\
\hline Market Mechanism & 3 & $3 \%$ \\
\hline Information Sheering & 3 & $3 \%$ \\
\hline Performance & 12 & $11 \%$ \\
\hline Salesforce & 6 & $6 \%$ \\
\hline Capacity & 6 & $6 \%$ \\
\hline Open Source & 9 & $8 \%$ \\
\hline Data Center & 6 & $6 \%$ \\
\hline Security & 23 & $21 \%$ \\
\hline Accessing Grid & 8 & $7 \%$ \\
\hline Network & 7 & $7 \%$ \\
\hline Architecture & 9 & $8 \%$ \\
\hline Modeling, Simulation & 4 & $4 \%$ \\
\hline Database & 5 & $5 \%$ \\
\hline Application & 6 & $6 \%$ \\
\hline & 107 & \\
\hline & &
\end{tabular}


Depend on the table (1), we have been reviewed the rank of the directions; "security" has the first rank with $23 \%$ of the total random sample as shows in chart (1).

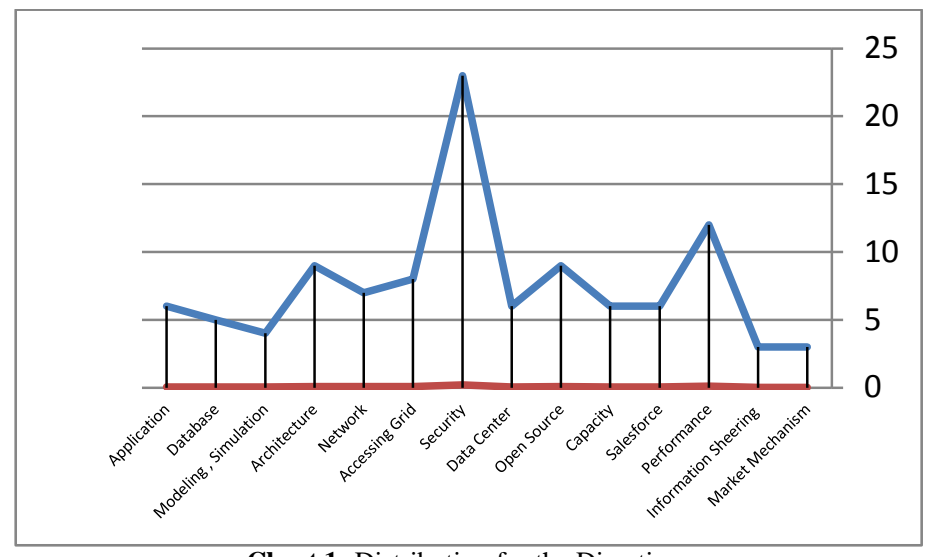

Chart 1: Distribution for the Directions

Table(2) illustrates the sort of the direction percentages from the smallest to the largest values.

Table 2: The sort from the lowest values for the directions percentage.

\begin{tabular}{|l|l|l|}
\hline Direction & No. & Per. \\
\hline Market Mechanism & 3 & $3 \%$ \\
\hline Information Sheering & 3 & $3 \%$ \\
\hline Modeling, Simulation & 4 & $4 \%$ \\
\hline Database & 5 & $5 \%$ \\
\hline Salesforce & 6 & $6 \%$ \\
\hline Capacity & 6 & $6 \%$ \\
\hline Data Center & 6 & $6 \%$ \\
\hline Application & 6 & $6 \%$ \\
\hline Network & 7 & $7 \%$ \\
\hline Accessing Grid & 8 & $7 \%$ \\
\hline Open Source & 9 & $8 \%$ \\
\hline Architecture & 9 & $8 \%$ \\
\hline Performance & 12 & $11 \%$ \\
\hline Security & 23 & $21 \%$ \\
\hline & 107 & \\
\hline & &
\end{tabular}

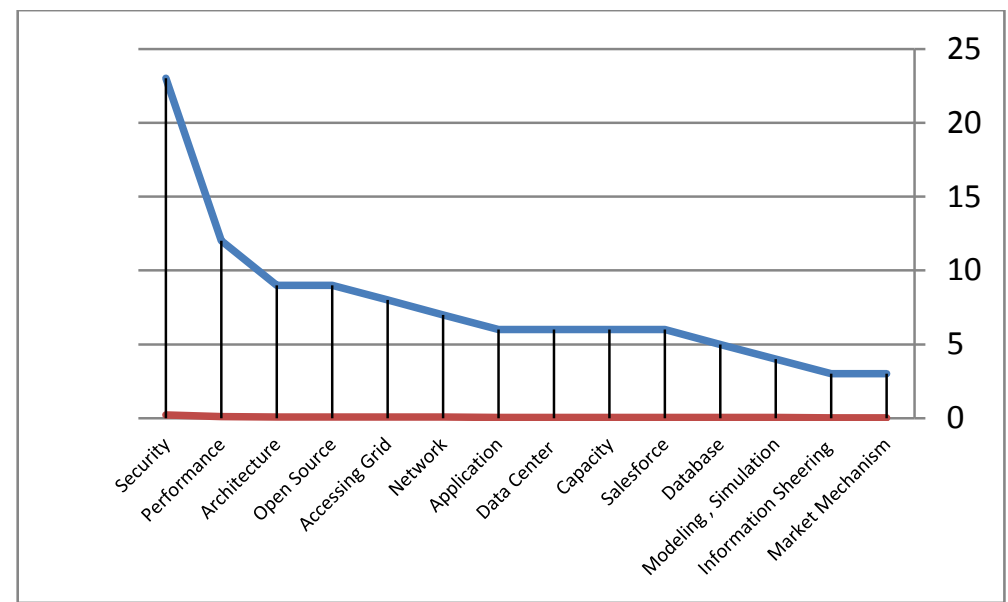

Chart 2:The Increasing Values for the Directions Percentage.

\section{Reviews on Cloud Computing Direction}

In order to illustrate the frequency of the selected random articles for the cloud computing reviews, table (3) shows the distribution for (102) articles those who are published for decade period of time (2003-2013). 
Table 3:Articles Distribution form (2003-2013)

\begin{tabular}{|l|l|l|}
\hline No & Year & Fre. \\
\hline 1 & 2003 & 3 \\
\hline 2 & 2004 & 2 \\
\hline 3 & 2006 & 2 \\
\hline 4 & 2007 & 2 \\
\hline 5 & 2008 & 10 \\
\hline 6 & 2009 & 23 \\
\hline 7 & 2010 & 18 \\
\hline 8 & 2011 & 10 \\
\hline 9 & 2012 & 8 \\
\hline 10 & 2013 & 24 \\
\hline & & 102 \\
\hline
\end{tabular}

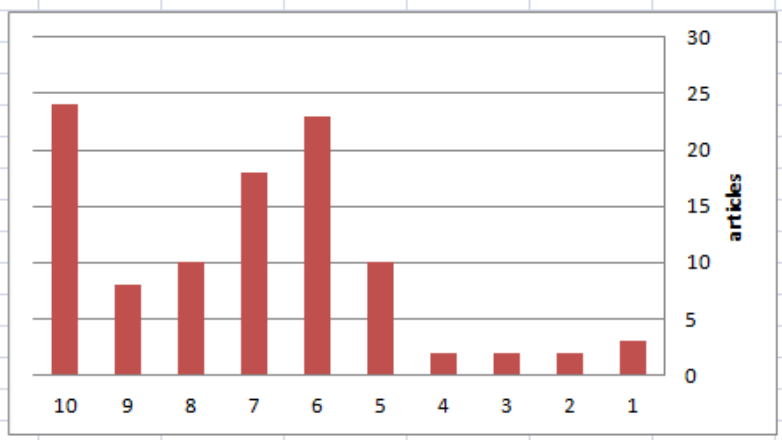

Chart 3: Articles for Decade of Time (2003-2013)

Chart (3) shows the most citation for the "cloud computing" key word, for the random sample. In the 2013 were about 24 articles. The second rank was in 2009 about 23.

\section{Conclusion}

Cloud services are increasing day to day, which means its future is very bright. Cloud services will eliminate the need to install and manage client rich applications and further its scope in all the private sector would increase thus it would help the company to reduce high-cost infrastructure and maintenance cost.

In this paper by analysis the direction papers on cloud computing, we build a model of review on the reviews direction by using a system dynamics methodology analysis. Looking back further to the papers direction will argue the papers' directions for the researcher. On the other hand, we argue that some new fundamentals on cloud computing need for issues in the future.

In this paper by analyzing of distribution frequency of the articles, it has been founded that the citation for the "cloud computing" increasing day after day.

In this paper it has been founded that publishing in the reliable journals make the citation easier, faster and dependable.

\section{References}

[1] Brototi Mondala,*, Kousik Dasguptaa, Paramartha Duttab, Load Balancing in Cloud Computing using Stochastic Hill Climbing-A Soft Computing Approach, 2012.

[2] Abdul Nasir Khana, *, M.L. Mat Kiah a, Samee U. Khanb, Sajjad A. Madanic, towards secure mobile cloud computing: A survey, 2012.

[3] Fa-Chang Chenga, Wen-Hsing Laib*, The Impact of Cloud Computing Technology on Legal Infrastructure within Internet-Focusing on the Protection of Information Privacy, 2012.

[4] Jaeger PT, Lin J, Grimes JM. Cloud computing and information policy: computing in a policy cloud? Journal of InformationTechnology and Politics 2008, 5(3): 269 - 283. http://dx.doi.org/10.1080/19331680802425479.

[5] Dawei Suna,*, Guiran Changb, Lina Suna and Xingwei Wanga, Surveying and Analyzing Security, Privacy and Trust Issues in Cloud Computing Environments,2013.

[6] Jie Tao, Holger Marten; David Kramer, Wolfgang Karl, An Intuitive Framework for Accessing Computing Clouds, 2011.

[7] Asli Deniz Helvacioglu Kuyucu, the Playground of Cloud Computing in Turkey, 2011.

[8] Ben Martini*, Kim-Kwang Raymond Choo, An integrated conceptual digital forensic framework for cloud computing, 2012.

[9] Gaofeng Zhang a, Yun Yanga, Jinjun Chen b, A historical probability based noise generation strategy for privacy protection in cloud computing, 2012.

[10] Mell P, Grance T. The NIST Definition of Cloud Computing. Communications of the ACM; 2010; 53(6):50

[11] Chow R, Golle P, Jakobsson M, Shi E, Staddon J, Masuoka R. et al. Controlling data in the cloud: outsourcing computation without outsourcing control. CCSW 2009. http://dx.doi.org/10.1145/1655008.1655020.

[12] Andrzej Goscinski _, Michael Brock, toward dynamic and attribute based publication, discovery and selection for cloud computing, 2010. 
[13] Arnon Rosenthal, Peter Mork *, Maya Hao Li, Jean Stanford, David Koester, Patti Reynolds, and Cloud computing: A new business paradigm for biomedical information sharing, 2009.

[14] Chang Liu a,*, Xuyun Zhanga, Chi Yangb, Jinjun Chena, CCBKE - Session key negotiation for fast and secure scheduling of scientific applications in cloud computing.

[15] Daniele Angeli, Enrico Masala, A cost-effective cloud computing framework for accelerating multimedia communication simulations, 2012.

[16] Dimitrios Zissis , Dimitrios Lekkas, Securing e-Government and e-Voting with an open cloud computing architecture,2011.

[17] Dimitrios Zissis *, Dimitrios Lekkas, Addressing cloud computing security issues, 2012

[18] Huaglory Tianfielda, ,, Rainer Unlandb , Towards autonomic computing systems, 2012.

[19] Ismail Ari $\Uparrow$, Nitel Muhtaroglu, Design and implementation of a cloud computing service for finite element analysis, 2011.

[20] K. Jorissen *, F.D. Vila, J.J. Rehr, A high performance scientific cloud computing environment for materials Simulations, 2012.

[21] Laboratoire LICEF, T616-universit6, Universit6 du Qu6becm, Application of Artificial Intelligence Techniques to Computer Network Topology Design, 2012.

[22] LICEF Laboratory, T\&universit . Giversit\& du Q\&bee, 1001, rue Sherbrooke Est, 4e Ptage, Case Postale 5250, Succ. C,Mont\&al, Q\&bec, Canada, H2L 4L5, An artificial intelligence approach for generating assembly sequences in CAD/CAM, 1995.

[23] M. Mackay a, T. Baker b, A. Al-Yasiri c, Security-oriented cloud computing platform for critical Infrastructures, 2012.

[24] Md. Tanzim Khorshed, A.B.M. Shawkat Ali *, Saleh A. Wasimi, A survey on gaps, threat remediation challenges and some thoughts for proactive attack detection in cloud computing, 2012.

[25] Nabil Sultan, Cloud computing for education: A new dawn, 2010

[26] Nir Kshetri, Privacy and security issues in cloud computing: The role of institutions and institutional evolution, 2013.

[27] Niroshinie Fernando *, Seng W. Loke *, Wenny Rahayu, Mobile cloud computing: A survey, 2013.

[28] S. Subashini n, V.Kavitha, A survey on security issues in service delivery models of cloud computing, 2012.

[29] Saurabh Kumar Garg a,*, Steve Versteeg b, Rajkumar Buyyaa, A framework for ranking of cloud computing services, 2013.

[30] Sean Marston a, Zhi Li a, Subhajyoti Bandyopadhyay, Juheng Zhang a, Anand Ghalsasi b, Cloud computing - the business perspective, 2011 .

[31] Shun-Sheng Wang, Kuo-Qin Yan *, Shu-Ching Wang, Achieving efficient agreement within a dual-failure cloud-computing environment, 2011.

[32] Slaven Brumec,NevenVrček, Cost effectiveness of commercial computing clouds, 2012.

[33] Subhas Chandra Misra *, Arka Mondal1, Identification of a company's suitability for the adoption of cloud computing and modelling its corresponding Return on Investment, 2011.

[34] R.Buyya, C. Yeo, S.Venugopal, J.Broberg, I.Brandic, Cloud computing and emerging it platforms: Vision, hype, and reality fordelivering computing as the 5th utility, in: Future Generation Computer Systems, vo1.25, 2009, pp. 599616.http://dx.doi.org/10.1016/j.future.2008.12.001

[35] G. Boss, P. Malladi, D. Quan, L. Legregn, Cloud computing, in: High Performance On Demand Solutions (HiPODS), IBM, 2007.

[36] R. R.Buyya, R.Ranjan, Intercloud: Utility-oriented federation of cloud computing environments for scaling of application services,in: ICA3PP 2010, Part I, LNCS 6081., 2010, pp. 13-31.

[37] T. R.Armstrong, D.Hensgen, The relative performance of various mapping algorithms is independent of sizable variances in runtimepredictions, in: 7th IEEE Heterogeneous Computing Workshop (HCW '98), 1998, pp. 7987.http://dx.doi.org/10.1109/HCW.1998.666547.

[38] A. Vouk, Cloud computing- issues, research and implementations, in: Information Technology Interfaces, 2008, pp. 31-40.

[39] Tuncay Ercan, Effective use of cloud computing in educational institutions, 2010.

[40] Weiwei Lina,*, James Z. Wangb, Chen Liangc, Deyu Qia, A Threshold-based Dynamic Resource Allocation Scheme for Cloud Computing,2012.

[41] Xun $\mathrm{Xu}$ n, from cloud computing to cloud manufacturing.Christine W. Chana, *, Guo H. Huangb, Artificial intelligence for management and control of pollution minimization and mitigation processes, 2012.

[42] R.N.Calheiros, R. Ranjan, A. Beloglazov, C. Rose, R. Buyya, Cloudsim: A toolkit for modeling and simulation of cloud computing environments and evaluation of resource provisioning algorithms, in: Software: Practice and Experience (SPE), Volume 41, Number 1, ISSN: 0038-0644, Wiley Press, New York, USA., 2011, pp. 23-50.

[43] A.M. Alakeel, A guide to dynamic load balancing in distributed computer systems, in: IJCSNS International Journal of Computer Science and Network Security, VOL.10No.6, 2010, pp. 153-160.

[44] S. Russell, P. Norvig, Artificial intelligence: A modern approach 3/e, in: Pearson Publication, ISBN-10: 0136042597, 2010.

[45] Mell P, Grance T. The NIST definition of cloud computing. National Institute of Standards and Technology 2009.

[46] Ruiter J, Warnier M. Privacy regulations for cloud computing - compliance and implementation in theory and practice. SPCC 2010.

[47] Rimal BP, Choi E, Lumb I. A taxonomy and survey of cloud computing systems. International Joint Conference on INC, IMS and IDC 2009, $44-51$.

[48] Catteddu D, Hogben G. Cloud computing: benefits, risks and recommendations for information security. Technical Report.European Network and Information Security Agency, 2009.

[49] Ristenpart T, Tromer E, Shacham H, Savage S. Hey, you, get off of my cloud: exploring information leakage in third-party compute clouds. CCS 2009.http://dx.doi.org/10.1145/1653662.1653687.

[50] Soghoian, C. Caught in the cloud: privacy, encryption, and government back doors in the Web 2.0 Era, J on Telecomm \& High Tech L 2009, 8: $359-424$

[51] Kant K, Iyer R, Mohapatra P. Architectural impact of secure socket layer on Internet servers. Int'l Conf on Computer Design 2000, 714.http://dx.doi.org/10.1109/ICCD.2000.878263

[52] Gentry C. Fully homomorphic encryption using ideal lattices. ACM Symposium on Theory of Computing 2009, 169178.http://dx.doi.org/10.1145/1536414.1536440.

[53] Cooney M. IBM touts encryption innovation. Computer World 2009, June 25.

[54] Schneier B. Homomorphic encryption breakthrough. Schneier on Security 2009, July 9.

[55] Pearson S, Shen Y, Mowbray M. A privacy manager for cloud computing. CloudCom 2009.http://dx.doi.org/10.1007/978-3-642-10665-1 9.

[56] Michael Armbrust, Armando Fox, Rean Griffith and et al. Above the Clouds: A Berkeley View of Cloud Computing [EB/OL], http://www.eecs.berkeley.edu/Pubs/TechRpts/2009/EECS-2009-28.html, February 10, 2009.

[57] Rajkumar Buyya, Chee Shin Yeo, Srikumar Venugopal, James Broberg, and Ivona Brandic, Cloud Computing and Emerging IT Platforms: Vision, Hype, and Reality for Delivering Computing as the 5th Utility, Future Generation Computer Systems, Volume 25, And Number 6, Pages: 599-616, ISSN: 0167-739X, Elsevier Science, Amsterdam, The Netherlands, and June 2009.

[58] Rajkumar Buyya, Chee Shin Yeo, and Srikumar Venugopal, Market-Oriented Cloud Computing: Vision, Hype, and Reality for Delivering IT Services as Computing Utilities, Keynote Paper, Proceedings of the 10th IEEE International Conference on High Performance Computing and Communications, Sept. 25-27, 2008, Dalian, China. 
[59] Xindong You, Xianghua Xu, Jian Wan, Dongjin Yu, "RAS-M: Resource Allocation Strategy Based on Market Mechanism in Cloud Computing," chinagrid, pp.256-263, 2009 Fourth ChinaGrid Annual Conference, 2009.

[60] Hien Nguyen Van, Frédéric Dang Tran, Jean-Marc Menaud, "SLA-Aware Virtual Resource Management for Cloud Infrastructures," cit, vol. 1 , pp.357-362, 2009 Ninth IEEE International Conference on Computer and Information Technology, 2009. http://dx.doi.org/10.1109/CIT.2009.109.

[61] P radeep Kumar,Vivek Kumar Segal, Durg Singh Chauhan, "Effective Ways of Secure, Private and Trusted Computing", International Journal of Computer Science Issues (IJCSI), vol8, Issues3,No2,May2011,pp412-421.

[62] Hien Nguyen Van, Frederic Dang Tran, Jean-Marc Menaud, "Autonomic virtual resource management for service hosting platforms," icsecloud, pp.1-8, 2009 ICSE Workshop on Software Engineering Challenges of Cloud Computing, 2009

[63] F. Hermenier, X. Lorca, J.-M. Menaud, G. Muller and J. Lawall. Entropy: a Consolidation Manager for Cluster. In proc.of the 2009 International Conferenceon Virtual Execution Environments (VEE’09), Mar.2009.

[64] Guiyi Wei, Athanasios V. Vasilakos, Yao Zheng and Naixue Xiong. A game-theoretic method of fair resource allocation for cloud computing services. The Journal of Supercomputing,Volume 54, Number 2, 252-269, Nov.2010.http://dx.doi.org/10.1007/s11227-009-0318-1.

[65] Rodrigo N. Calheiros, Rajiv Ranjan, Cesar A. F. De Rose, and Rajkumar Buyya, "CloudSim: A Novel Framework for modeling and Simulation of Cloud Computing Infrastructures and Services", 2009.

[66] Rahul Singh, Upendra Sharma, Emmanuel Cecchet and Prashant Shenoy. Autonomic Mix-Aware Provisioning for Non-Stationary Data Center Workloads, Proceedings of the 7th IEEE International Conference on Autonomic Computing and Communications (ICAC), Washington, DC, USA, June 7-11, 2010.http://dx.doi.org/10.1145/1809049.1809053.

[67] J. Hellerstein, F. Zhang, and P. Shahabuddin. An Approach to Predictive Detection for Service Management. In Proceedings of the IEEE Intl. Conf. on Systems and Network Management, 1999.http://dx.doi.org/10.1109/INM.1999.770691.

[68] D. Menasce and F. Ribeiro. In search of invariants for e-business workloads. In Proceedings of the 2nd ACM conference on Electronic Commerce, pages 56-65, 2000.http://dx.doi.org/10.1145/352871.352878.

[69] Jakarta Apache. JMeter. http://jakarta.apache.org/jmeter/index.html,2010.12.

[70] H. Lim, S. Babu, J. Chase, and S. Parekh. Automated Control in Cloud Computing: Challenges and Opportunities, 2009.

[71] Proc. of the First Workshop on Automated Control for Datacenters and Clouds, June 2009:13-18.

[72] Foster I, Zhao Y, Raicu I, Lu, S. Cloud Computing and Grid Computing 360-degree compared. Proceedings of the GridComputing Environments Workshop, GCE 2008; IEEE Press, Nov. 2008, 1-10.http://dx.doi.org/10.1109/GCE.2008.4738445.

[73] Buyya R, Chee Shin Y, Venugopal S, Broberg J, Brandic I. Cloud computing and emerging IT platforms: vision, hype, andreality for delivering computing as the 5th utility. Future Generation Computer Systems; 2009; 25(6):599616.http://dx.doi.org/10.1016/j.future.2008.12.001.

[74] Armbrust M, Fox A, Griffith R, Joseph A D, Katz R, Konwinski A, Lee G, Patterson D, Rabkin A, Stoica I, Zaharia M. AView of Cloud Computing. Communications of the ACM; 2010; 53(4):50-58.http://dx.doi.org/10.1145/1721654.1721672.

[75] Paquette S, Jaeger P T, Wilson S C. Identifying the security risks associated with governmental use of cloud computing.Government Information Quarterly; 2010;27(3):245-253.http://dx.doi.org/10.1016/j.giq.2010.01.002.

[76] Subashini S, Kavitha V. A survey on security issues in service delivery models of cloud computing. Journal of Network andComputer Applications; 2011; 34(1):1-11.http://dx.doi.org/10.1016/j.jnca.2010.07.006.

[77] Vaquero L M, Rodero-Merino L, Morán D. Locking the sky: A survey on IaaS cloud security. Computing; 2011; 91(1):93118.http://dx.doi.org/10.1007/s00607-010-0140-x.

[78] B. Bazeer Ahamed, S. Syed Sabir Mohamed, "Implementation of Trusted Computing Technologies in Cloud Computing" International Journal of Research and Reviews in Information Sciences(IJRRIS),Vol. 1, No. 1, March 2011.pp 7-9

[79] Pearson S, Benameur A. Privacy, security and trust issues arising from cloud computing. Proceedings of the 2nd IEEEInternational Conference on Cloud Computing Technology and Science, CloudCom 2010; IEEE Press, Nov. 2010, 693702.http://dx.doi.org/10.1109/CloudCom.2010.66.

[80] Ahamed S I, Haque M M, Endadul Hoque M, Rahman F, Talukder N. Design, analysis, and deployment of omnipresentformal trust model (FTM) with trust bootstrapping for pervasive environments. Journal of Systems and Software; 2010; 83 (2):253270.http://dx.doi.org/10.1016/j.jss.2009.09.040.

[81] Karaoglanoglou K, Karatza H. Resource discovery in a Grid system: Directing requests to trustworthy virtual organizationsbased on global trust values. Journal of Systems and Software; 2011; 84(3):465-478.http://dx.doi.org/10.1016/j.jss.2010.10.043.

[82] Takabi H, Joshi J B D, Ahn G. Security and privacy challenges in cloud computing environments. IEEE Security \& Privacy; 2010;8(6):2431.http://dx.doi.org/10.1109/MSP.2010.186.

[83] Sangroya A, Kumar S, Dhok J, Varma V. Towards analyzing data security risks in cloud computing environments.Communications in Computer and Information Science; 2010;54:255-265.http://dx.doi.org/10.1007/978-3-642-12035-0_25.

[84] Algirdas A, Jean-Claude L, Brian R, Carl L. Basic concepts and taxonomy of dependable and secure computing. IEEETransactions on Dependable and Secure Computing; 2004; 1 (1):11-33.http://dx.doi.org/10.1109/TDSC.2004.2.

[85] Jason Reid Juan M. González Nieto Ed Dawson, "Privacy and Trusted Computing", Proceedings of the 14th International Workshop on Database and Expert Systems Applications, IEEE, 2003

[86] Tchifilionova V. Security and privacy implications of cloud computing - Lost in the cloud. Proceedings of the IFIP WG 11.4 International Workshop on Open Research Problems in Network Security, iNetSec 2010; Springer Verlag Press, Mar. 2010, 149-158.

[87] Krumm J. A survey of computational location privacy. Personal and Ubiquitous Computing; 2009; 13 (6):291399.http://dx.doi.org/10.1007/s00779-008-0212-5.

[88] Shekarpour S, Katebi S D. Modeling and evaluation of trust with an extension in semantic web. Journal of Web Semantics; 2010; 8(1):2636.http://dx.doi.org/10.1016/j.websem.2009.11.003.

[89] Iltaf N, Hussain M, Kamran F. A mathematical approach towards trust based security in pervasive computing environment, 2009.

[90] Proceedings of the Third International Conference and Workshops, ISA 2009; IEEE Press, Jun. 2009, 702-711.

[91] H. Kornmayer et al., gEclipse- An Integrated, Grid Enabled Workbench Tool for Grid Application Users, Grid Developers and Grid Operators based on the Eclipse Platform, in: Proceedings of the 2nd Austrian Grid Symposium, Innsbruck, Austria, 2006, project web:http://www.geclipse.eu/.

[92] Xiao-Yong Li , Li-Tao Zhou ,Yong Shi and Yu Guo, "A trusted computing environment model in cloud architecture", International Conference on Machine Learning and Cybernetics (ICMLC), July 2010, Volume 6, pp. 28432848http://dx.doi.org/10.1109/ICMLC.2010.5580769.

[93] M. Malawski1, T. Bartynski, M. Bubak, a Tool for Building Collaborative Applications by Invocation of Grid Operations, in: ComputationalScience - ICCS 2008, Vol. 5103 of LNCS, 2008, pp. 243-252.

[94] OMII-Europe, The OMII-Europe Project, http://www.omii-europe.com/index.html.

[95] Google, Google App Engine, http://code.google.com/intl/de-DE/appengine/.

[96] Balakrishnan.S,Saranya.G,Shobana.S,Karthikeyan.S, "Introducing Effective Third Party Auditing (TPA) for Data Storage Security in Cloud". International Journal of Computer Science and Technology Vol. 2, Issue 2, June 2011, pp398-400.

[97] Microsoft, Windows Azure Platform, http://www.zimory.com/. 
[98] Salesforce, Salesforce Software, http://www.salesforce.com/.

[99] OpSource Inc., OpSource Cloud, ttps://www.opsourcecloud.net/.

[100] Zimory, Zimory Public Cloud,http://www.microsoft.com/windowsazure/.

[101] K. Keahey ET. al., Science Clouds: Early Experiences in Cloud Computing for Scientific Applications, in: Proceedings of CCA-08, 2008.

[102] L. Wang, J. Tao, M. Kunze, Scientific Cloud Computing: Early Definition and Experience, in: Proceedings of the 2008 International Conferenceon High Performance Computing and Communications (HPCC 2008), 2008, pp. 825830.http://dx.doi.org/10.1109/HPCC.2008.38.

[103] D. Nurmi ET. al., The Eucalyptus Open-source Cloud Computing System, in: Proceedings of CCA-08, 2008

[104] B. Sotomayor ET. al., Capacity Leasing in Cloud Systems using the OpenNebula Engine, in: Proceedings of CCA-08, 2008.

[105] P.Wolniewicz et al., Accessing Grid computing resources with g-Eclipse platform, Computational Methods in Science and Technologie 13 (2)(2007) 131-141.http://dx.doi.org/10.12921/cmst.2007.13.02.131-141.

[106] Hadoop, Apache Hadoop MapReduce, http://hadoop.apache.org/.

[107] Xuan Zhang Wuwong, N. Hao Li Xuejie Zhang, "Information Security Risk Management Framework for the Cloud Computing Environments" IEEE 10th International Conference Computer and Information Technology (CIT), 2010, pp. 1328 - 1334. 